BNL-114710-2017-JA

File \# 95718

\title{
Imaging Nutrient Distribution in the Rhizosphere using FTIR Imaging
}

\author{
T. Victor, L. M. Miller \\ Submitted to: Analytical Chemistry \\ March 6, 2017 \\ Photon Sciences Department \\ Brookhaven National Laboratory \\ U.S. Department of Energy
USDOE Office of Science (SC),
Basic Energy Sciences (BES) (SC-22)
}

Notice: This manuscript has been authored by employees of Brookhaven Science Associates, LLC under Contract No. DE- SC0012704 with the U.S. Department of Energy. The publisher by accepting the

manuscript for publication acknowledges that the United States Government retains a non-exclusive, paid-up, irrevocable, world-wide license to publish or reproduce the published form of this manuscript, or allow others to do so, for United States Government purposes. 


\section{DISCLAIMER}

This report was prepared as an account of work sponsored by an agency of the United States Government. Neither the United States Government nor any agency thereof, nor any of their employees, nor any of their contractors, subcontractors, or their employees, makes any warranty, express or implied, or assumes any legal liability or responsibility for the accuracy, completeness, or any third party's use or the results of such use of any information, apparatus, product, or process disclosed, or represents that its use would not infringe privately owned rights. Reference herein to any specific commercial product, process, or service by trade name, trademark, manufacturer, or otherwise, does not necessarily constitute or imply its endorsement, recommendation, or favoring by the United States Government or any agency thereof or its contractors or subcontractors. The views and opinions of authors expressed herein do not necessarily state or reflect those of the United States Government or any agency thereof. 


\section{Imaging Nutrient Distribution in the Rhizosphere using FTIR Imaging}

Tiffany Victor, ${ }^{1}$ Natalie Delpratt, ${ }^{2}$ Sarah Beth Cseke, ${ }^{3}$ Lisa M. Miller, ${ }^{1,2}$ Leland James Cseke ${ }^{3 *}$

${ }^{1}$ Department of Chemistry, Stony Brook University, Stony Brook, New York 11794

${ }^{2}$ National Synchrotron Light Source II, Brookhaven National Laboratory, Upton, New York 11973

${ }^{3}$ Department of Biological Science, University of Alabama in Huntsville, Huntsville, Alabama 35899

${ }^{*}$ Corresponding author:

Leland J. Cseke, $\mathrm{PhD}$

Department of Biological Science

The University of Alabama in Huntsville

Huntsville, Alabama 35899

Phone: (256) 824-6774

Fax: (256) 824-6305

E-mail: csekel@uah.edu

Keywords: FTIR imaging, mycorrhizal fungi, rhizosphere, Phytagel, nutrient distribution 


\begin{abstract}
Symbiotic associations in the rhizosphere between plants and microorganisms lead to efficient changes in the distribution of nutrients that promote growth and development for each organism involved. Understanding these nutrient fluxes provides insight into the molecular dynamics involved in nutrient transport from one organism to the other. To study such nutrient flow, a new application of Fourier transform infrared imaging (FTIRI) was developed that entailed growing Populus tremulodes seedlings on a thin, nutrient-enriched Phytagel matrix that allows pixel to pixel measurement of the distribution of nutrients - in particular nitrate - in the rhizosphere. The FTIR spectra collected from ammonium nitrate in the matrix indicated the greatest changes in the spectra at $1340 \mathrm{~cm}^{-1}$ due to the asymmetric stretching vibrations of nitrate. To quantify the nitrate concentration in the rhizosphere of experimental plants, a calibration curve was generated that gave the nitrate concentration at each pixel in the chemical image. These images of the poplar rhizosphere showed evidence for symbiotic sharing of nutrients between the plant and the fungi, Laccaria bicolor, where the nitrate concentration was five times higher near mycorrhizal roots than further out into the rhizosphere. This suggested that nitrates are acquired and transported from the media toward the plant root by the fungi. Similarly, the sucrose used in the growth media as a carbon source was depleted around the fungi, suggesting its uptake and consumption by the system. This study is the first of its kind to visualize and quantify the nutrient availability associated with mycorrhizal interactions, indicating that FTIRI has the ability to monitor nutrient changes with other microorganisms in the rhizosphere as a key step for understanding nutrient flow processes in more diverse biological systems.
\end{abstract}




\section{Introduction}

The rhizosphere is an area surrounding the roots of plants that is most directly involved in nutrient uptake processes, typically considered to extend at least $2-5 \mathrm{~mm}$ away from the plant root. ${ }^{1,2}$ Within the rhizosphere, several important processes occur including changes in the nutrient distribution as a consequence of symbiotic interactions between plants and microbes. ${ }^{3}$ The understanding of these processes is essential to the prediction and utilization of the natural nutrient cycling processes that control sustainable plant growth and development.

Understanding changes in small, inorganic nutrients including nitrate, sulfate, and phosphate in the rhizosphere has been limited by the lack of suitable techniques to reliably detect nutrients both spatially and temporally. ${ }^{4}$ Traditionally, bulk mass spectrometry techniques have been used to examine nutrients including nitrogen, phosphorus, and carbon in the rhizosphere.

Unfortunately, the use of these techniques results in a loss of the spatially resolved information necessary to determine accurate nutrient flow and distribution due to the signal averaging between different nutrients in the system. ${ }^{4}$ Thus, to improve our understanding of spatial and temporal events associated with nutrient cycling, there is a need for new chemical visualization approaches that can accurately target and trace changes quantitatively in the availability of specific nutrient compounds.

More recently, X-ray Fluorescence microscopy (XFM) and Nanoscale Secondary Ion Mass Spectrometry (NanoSIMS) ${ }^{4}$ have been used to obtain spatially-resolved chemical information about nutrients in situ in the rhizosphere. In XFM, a focused X-ray beam is used to obtain energy resolved fluorescence spectra that identify the presence of trace elements for each position scanned. The use of XFM, especially coupled to a synchrotron source, provides sub-micron 
resolution, which translates into detection limits in the range sub-ppm of trace elements in the rhizosphere. ${ }^{6}$ However, XFM is unable to detect low-Z elements such as carbon and nitrogen.

The other method that has been employed in studying rhizosphere processes, NanoSIMS, is a specific type of secondary ion mass spectrometry instrument that has been designed for high lateral resolution (down to $50 \mathrm{~nm}$ ) imaging while still maintaining high mass resolution and high sensitivity (mg/kg range). ${ }^{7}$ However, it is difficult to analyze some elements because of poor secondary ion yield, the inability to quantify actual concentrations, and unobtainable chemical state information. ${ }^{7}$ Furthermore, the field of view is limited, which means that mapping large areas is relatively slow and such experiments must be done under ultra-high vacuum, which is not conducive to biological samples. ${ }^{7}$

Here, a new, complementary approach is introduced to address the aforementioned limitations by using Fourier transform infrared imaging (FTIRI) to image the distribution of nutrients in the rhizosphere of a woody plant. FTIRI provides an approach to simultaneously image and quantify cellular components, micro- and macronutrients, and exudates that are important to rhizosphere activity with high spatial resolution under environmentally-relevant conditions. The method is rapid and non-destructive, making it an excellent choice to reliably visualize and quantify nutrients in the rhizosphere on a sub-millimeter scale. It is particularly well-suited for measuring inorganic ions like nitrates, phosphates and sulfates because these polyatomic macronutrients exhibit characteristic infrared vibrational frequencies that are unique.

The objective of this study was to develop an FTIRI method to study nutrient distribution in an intact plant's rhizosphere. To accomplish this, a unique experimental model system was used to minimize system complexity and to permit precise manipulation of nutrient parameters. The system was comprised of a tree species, Populus tremuloides (quaking aspen), and the 
ectomycorrhizal fungus, Laccaria bicolor, grown in McCown Woody Plant Media (WPM). The plant growth media is composed of WPM nutrient salts and sucrose in a Phytagel matrix, allowing the two organisms to form fully mature mycorrhizal interactions between the fungi and tree roots. ${ }^{8,9}$ By using FTIRI, the nitrate and sucrose distribution in the poplar rhizosphere was measured simultaneously to gain insight into nutrient sharing in mycorrhizal plant-fungus interactions. This ability to visually monitor nutrient changes with other microorganisms in the rhizosphere is a key first step towards improving our understanding of the nutrient flow processes in more complex systems.

\section{Methods}

\section{Nutrient Media Preparation}

McCown's Woody Plant Media (WPM), a widely used culture media for tree species, was used for each experiment. ${ }^{10}$ The media solution was prepared by adding $2.4 \mathrm{~g} / \mathrm{L} \mathrm{McCown's}$ Woody Plant Media (WPM), $0.5 \mathrm{~g} / \mathrm{L} 2$-( $N$-morpholino) ethanesulfonic (MES), and $15 \mathrm{~g} / \mathrm{L} \mathrm{mM}$ sucrose to $1000 \mathrm{ml}$ of $\mathrm{ddH}_{2} \mathrm{O}$. For each experiment requiring plant growth, two types of media were required, (1) solid media used as the rhizosphere matrix and to directly supply nutrients to the trees, and (2) liquid media used to maintain nutrient supply and hydration of the system. The $\mathrm{pH}$ of the solution was then adjusted to 5.6 using a $1 \mathrm{M} \mathrm{KOH}$ solution. For the solid matrix, 8 g/L of Phytagel (Sigma Aldrich Phytagel, P8169) was added, and the media was autoclaved using a pre-set liquid cycle which lasted about 70 minutes. Once the cycle was complete, the media was placed into a sterile hood (SterilGARD III, The Baker Company, Sanford, ME) utilizing aseptic technique, and the temperature was monitored using a surface sterilized thermometer placed inside the media vessel. 
Slide preparation (dip-coating):

Preparation for dipping the slides was critical. Two hot plates were surface-sterilized in the hood and calibrated to maintain a media temperature at $50^{\circ} \mathrm{C}$ in order to provide an ideal thickness of media $(0.5 \mathrm{~mm})$ during slide preparation. A sterile stir bar provided the needed media circulation to maintain a uniform temperature throughout the media.

Infrared-reflective (low-e coated) glass microscope slides were used for all infrared imaging experiments (Kevley Technologies, Chesterland, OH, USA). To provide a sterile surface on which to adhere the plant media, slides were placed in aluminum foil packets, being careful not to let the coated side of the slides come into contact with the aluminum to prevent scratching. These packets were then autoclaved on a dry / kill cycle along with all glassware, stir bars, and forceps used in the remaining procedure. After autoclaving, the slides were placed at $80^{\circ} \mathrm{C}$ until use.

All dipping steps were conducted inside the sterile hood. The foil packet was removed from the freezer and placed on a bed of ice to keep the slides chilled. Slides were then dipped vertically into the freshly prepared plant media being held at $50^{\circ} \mathrm{C}$, held for one second, removed and then placed horizontally on the surface of a sterile Petri plate until the WPM media solidified ( $\sim 45$ minutes) (Figure 1). The media covered roughly $3 / 4$ of the slide. Slides were then placed vertically into $50 \mathrm{~mL}$ Falcon tubes containing $10 \mathrm{ml}$ of liquid WPM media in preparation for subsequent steps.

Poplar seed sterilization and growth

Populus tremuloides Michx seeds were obtained from the Canadian Natural Resources, National Tree Seed Center, P.O. Box 4000, Fredericton NB Canada, E3B 5P7 
(http://www.atl.cfs.nrcan.gc.ca/seedcentre/seed-center-e.htm). Seed Lot No. 9910008.0 was removed from $-20^{\circ} \mathrm{C}$ and incubated overnight at $4^{\circ} \mathrm{C}$ before they were transferred to a microcentrifuge tube and surface-sterilized using $1 \mathrm{ml}$ of sterilization solution $(10 \%$ commercial bleach, $250 \mu \mathrm{g} / 1$ Tween-20 and sterile water) for 5 minutes. The surface sterilization solution was removed, and the seeds were subsequently washed four times with sterile distilled water. ${ }^{11}$

For germination, the poplar seeds were suspended in $0.1 \%$ sterile agarose and left for two days in the dark at room temperature. Seeds showing signs of germination were individually embedded in the gelled media near the top of each slide using a sterilized spatula and forceps. The prepared slides were then placed back into each $50 \mathrm{~mL}$ Falcon tube containing $10 \mathrm{~mL}$ of liquid media. All slides were incubated at room temperature overnight in the dark. Subsequently, the tree seedlings were transferred to a growth chamber at $22{ }^{\circ} \mathrm{C}, 60 \%$ humidity, and running a $16 \mathrm{~h}$ day $/ 8 \mathrm{~h}$ night regime for 7 weeks to allow growth and development.

\section{Laccaria bicolor strain and culture conditions}

For samples incubated with Laccaria bicolor, free-living mycelium of the Orton strain S238N (dikaryotic) were sub-cultured and maintained on Modified Melin Norkan's (MMN) medium at $20^{\circ} \mathrm{C}$ in the dark. For inoculation experiments, L. bicolor mycelia were moved to the slides containing WPM when the P. tremuloides seedlings were 10 days old. This was accomplished by cutting the MMN media into $\sim 2 \mathrm{~mm}$ squares under sterile conditions in the hood. These fungal "plugs" were then placed face down on each slide next to the roots of each seedling using aseptic technique. After inoculation, the seedlings were returned to the growth chamber for the reminder of the 7 weeks to allow symbiotic interactions to establish and mycorrhizal roots to form during tree development.

\section{FTIRI spectroscopy and imaging}


Once the plants were 7 weeks old, the samples were dehydrated in an incubator at $60^{\circ} \mathrm{C}$ for 75 minutes and allowed to cool to room temperature. Under these conditions, the slides were observed to dry uniformly within 15 minutes; however, an additional hour of gentle drying was used to drive off any residual moisture. Slides were stored in a dark, desiccated environment until FTIRI was performed. All FTIR images were obtained from the dried samples using a Spectrum Spotlight 400 FTIR Imaging System (PerkinElmer) in transflectance mode. Spectra were collected in the mid-IR region from 4000 to $750 \mathrm{~cm}^{-1}$ using a 16 pixel MCT (Mercury Cadmium Telluride) array detector with a $25 \mu \mathrm{m}$ pixel size. The spectral resolution was $8 \mathrm{~cm}^{-1}$ and 16 scans per spectrum were collected.

FTIR spectra were also collected from the individual components of the media, i.e. sucrose, WPM, Phytagel, and ammonium nitrate. Spectra were collected in the mid-IR region from $4000-750 \mathrm{~cm}^{-1}$ using a spectral resolution of $4 \mathrm{~cm}^{-1}$ and 8 scans per spectrum. Spectra of the individual components were collected using Beamline U2B at the National Synchrotron Light Source at Brookhaven National Laboratory.

\section{Results}

Poplar plant growth and development

After seed sterilization, viable $P$. tremuloides seeds began to germinate after approximately 2 days in the dark. At that time, the newly germinated seeds were transferred to prepared IR reflective slides under sterile conditions and allowed to develop at $22{ }^{\circ} \mathrm{C}$ with a 16 hour light period. After 10 days of development, lateral roots began to form ${ }^{8}$ at which point trees requiring interaction with Laccaria were inoculated with small MMN media plugs containing 
fungal hyphae. Both the free-living seedlings and those inoculated with Laccaria were then allowed to develop for a total of 7 weeks. At week 3 , a clear fungal hyphae network could be seen growing along the developing roots of inoculated trees. By week 5 , the primary roots of the seedlings had grown to a point where they were entering the liquid WPM media at the base of each tube, and the formation of mychorrhizal lateral roots was clearly visible. At week 7 , these interactions had matured, and the slides were dehydrated to remove water from the slides. Water is highly IR absorptive, and even a slight amount of water in the media limits the quality of FTIR spectra that can be obtained.

\section{FTIR spectra of media components}

The first step in characterizing the poplar rhizosphere with FTIRI was to determine the individual spectral contributions of the media to the FTIR images. The components of the plant media include Phytagel, WPM, sucrose and MES (Table S1). Phytagel, while providing a visibly clear gel, contributes a number of absorption features in the overall FTIR spectra (Figure 2). Phytagel is a high strength gelling ingredient in the growth media, consisting of a tetrasaccharide repeating unit of two glucose residues, rhamnose and glucoronic acid. Phytagel has characteristic infrared vibrations that include an $\mathrm{OH}$ stretch $\left(3381 \mathrm{~cm}^{-1}\right)$, asymmetric $\mathrm{C}-\mathrm{H}$ stretch $\left(2930 \mathrm{~cm}^{-1}\right)$, and asymmetric COO${ }^{-}$stretch $\left(1610 \mathrm{~cm}^{-1}\right) .{ }^{12}$ The Phytagel polymer absorbs strongly from $1200-950 \mathrm{~cm}^{-1}$ and has a number of overlapping peaks. The bands in the $905-1155 \mathrm{~cm}^{-1}$ region are assigned to $\mathrm{C}-\mathrm{O}$ and $\mathrm{C}-\mathrm{C}$ stretching modes ${ }^{13}$ and those around $1200-1155 \mathrm{~cm}^{-1}$ have contributions from the bending modes of O-C-H, C-C-H and C-O-H. ${ }^{13} \cdot{ }^{14}$ The asymmetric carboxylate stretch at $1610 \mathrm{~cm}^{-1}$ does not overlap with the spectral features from other components in this system, making it the signature peak for quantifying Phytagel in these experiments. 
WPM contains all of the macronutrients, micronutrients, and vitamins needed for woody plant tissue culture as described by Lloyd and McCown. ${ }^{10,11}$ The most noticeable infrared vibrations in the WPM spectrum occur between $1500-1000 \mathrm{~cm}^{-1}$ (Figure 2) and can be attributed to the phosphates $\left(1092 \mathrm{~cm}^{-1}\right),{ }^{15}$ nitrates $\left(1349 \mathrm{~cm}^{-1}\right),{ }^{16}$ and sulfates $\left(1198 \mathrm{~cm}^{-1}\right),{ }^{17}$ found in the WPM salt mix.

Sucrose was added to the media to facilitate plant growth. Its polysaccharide structure is revealed by the broad peak at $\sim 1000 \mathrm{~cm}^{-1}$. In addition, sucrose has a distinguishable infrared vibration at $925 \mathrm{~cm}^{-1}$ that corresponds to its glycosidic links, ${ }^{12,14}$ which has the least overlap with other media components. Thus, the combined spectrum of the major components in the media shows that the region from $1500-1000 \mathrm{~cm}^{-1}$ has a large overlap of many spectral components, ${ }^{18}$ but the Phytagel and sucrose can be most easily identified through their isolated vibrations at $1610 \mathrm{~cm}^{-1}$ and $925 \mathrm{~cm}^{-1}$, respectively. ${ }^{12,15}$

\section{Quantification of nutrient concentration}

In FTIR absorption spectroscopy, it is well established that absorbance is proportional to concentration as defined by the Beer-Lambert Law $(A=\varepsilon b c)$. In particular, the concentration of nitrate in the poplar rhizosphere was determined using the Beer-Lambert Law $(\mathrm{A}=\varepsilon \mathrm{bc})$, where A is the FTIR absorbance at a particular wavelength, $\varepsilon$ is the absorption coefficient at a particular wavelength, $\mathrm{b}$ is the sample thickness, and $\mathrm{c}$ is the sample concentration. However, as is noted in the Beer-Lambert Law, knowledge of the absorption coefficient and sample thickness must be known in order to determine the nutrient concentration. To calculate the Phytagel thickness, the fringing effect of thin films was applied (see Supplemental Information). The average Phytagel thickness using this method was found to be $2.4 \pm 0.07 \mu \mathrm{m}$. The error of the technique was determined based on the $3 \%$ error obtained from a control polyimide film. 
The absorption coefficient was calculated for the asymmetric carboxylate peak at 1610 $\mathrm{cm}^{-1}$ because it has very little overlap with the spectra of the micro/macro nutrients or plant components. By using the calculated Phytagel thickness of $2.4 \mu \mathrm{m}$, a Phytagel concentration of $0.008 \mathrm{mM}$, and a measured absorbance of 0.3 for the asymmetric carboxylate peak at $1610 \mathrm{~cm}^{-1}$, the Beer-Lambert Law showed that the extinction coefficient for Phytagel at $1610 \mathrm{~cm}^{-1}$ to be $1.56 \times 10^{5} \mathrm{~cm}^{-1} \mathrm{mM}^{-1}$.

\section{Nitrogen detection}

Assignment of nitrate spectral features: In order to quantify the nutrients in the rhizosphere, nitrogen in this case, the spectral contributions of ammonium $\left(\mathrm{NH}^{+}\right)$and nitrate $\left(\mathrm{NO}^{-}\right)$were first identified. The FTIR spectrum of ${ }^{14} \mathrm{NH}_{4}{ }^{14} \mathrm{NO}_{3}$ can be seen in Figure 3, where two strong bands were observed at 1340 and $1432 \mathrm{~cm}^{-1}$. In order to assign these peaks, isotopically-substituted $\mathrm{NH}_{4} \mathrm{NO}_{3}$ was examined. From the FTIR spectrum of ${ }^{14} \mathrm{NH}_{4}{ }^{15} \mathrm{NO}_{3}$, it can be seen that the $1340 \mathrm{~cm}^{-1}$ band shifts to $1305 \mathrm{~cm}^{-1}$ due to the larger reduced mass of the heavier ${ }^{15} \mathrm{NO}_{3}$ isotope, indicating that this peak is assigned to the $\mathrm{NO}_{3}$ stretching vibration. Also, the ${ }^{14} \mathrm{NO}_{3}$ bending vibration was observed at $827 \mathrm{~cm}^{-1}$, which shifted to $806 \mathrm{~cm}^{-1}$ upon ${ }^{15} \mathrm{NO}_{3}$ substitution, confirming the assignment (Figure 3 inset). A similar analysis for the ${ }^{14} \mathrm{NH}_{4}$ bending vibration mode, which was found at $1432 \mathrm{~cm}^{-1}$, was shown to shift to $1425 \mathrm{~cm}^{-1}$ for ${ }^{15} \mathrm{NH}_{4}$.

Calibration curve: Once the spectral features of nitrate were determined, quantification of the nitrate content in the rhizosphere was done using a calibration curve of nitrate absorbance intensity versus nitrate concentration. To generate the calibration curve, infrared-reflective slides were dip-coated in Phytagel media that contained 7, 10, 15 or $20 \mathrm{mM}$ ammonium nitrate and allowed to dry. For each slide, twenty FTIR spectra were collected in transflectance mode at 
different locations on the slide. The slide preparation process was repeated three times. An average spectrum was calculated for each ammonium nitrate concentration from the 60 spectra. Each average spectrum was then baseline-corrected using points at $3900,1800,1510,1210$, and $790 \mathrm{~cm}^{-1}$. The four baselined spectra were normalized such that the Phytagel carboxylate peak at $1610 \mathrm{~cm}^{-1}$ had an absorbance value of 0.2 (Figure 4A). The absorbance intensity at the nitrate stretching vibration $\left(1340 \mathrm{~cm}^{-1}\right)$ was then plotted against the nitrate concentration to yield the calibration curve in Figure 4B.

Nutrient uniformity in the absence of plant tissue

To confirm a uniform nutrient distribution on the Phytagel slide in the absence of a plant, FTIR images were collected from the control slides described above. Specifically, FTIR maps measuring $1 \mathrm{~mm}^{2}$ (40x40 pixels) were collected at five different regions on each slide. An integration profile of the nitrate peak was generated by calculating the peak height at $1340 \mathrm{~cm}^{-1}$ using a linear baseline from $1505-1255 \mathrm{~cm}^{-1}$. Similarly, an integration profile of the Phytagel peak was generated by calculating the peak height at $1610 \mathrm{~cm}^{-1}$ using a linear baseline from 1775 $-1545 \mathrm{~cm}^{-1}$. In order to account for Phytagel thickness variations in the sample, a normalization matrix was generated that yielded a constant Phytagel intensity of 0.2 at each pixel. Then the nitrate image was normalized to thickness by applying the normalization matrix. Finally, the nitrate concentration was calculated from the normalized nitrate image using the calibration curve from Figure 4B. An average percent difference of $4 \%$ was calculated across 20 maps. Thus, for a standard Phytagel slide made with $7 \mathrm{mM}$ nitrate, the nitrate concentration varied from $7.0 \pm 0.28 \mathrm{mM}$, which is negligible compared to the plant's influence on the media.

Nutrient distribution in the presence of plant tissue 
The visible and infrared images of a 7 week old Poplar plant lateral root grown with Laccaria is shown in Figure 5. The visible image clearly shows the root colonization by the fungi. L. bicolor is an ectomycorrhizal fungi that forms a dense hyphal covering (fungal sheath or mantel) over the root from which hyphae grow into the intercellular spaces forming a net of internal hyphae (i.e. the Hartig net) around the root cortex cells, but do not penetrate the cell walls ${ }^{19},{ }^{2}$. This hyphal sheath forms an extension of the plant roots to increase the surface area of soil explored ${ }^{2}$ called the extra-radical mycelium (ERM). The fungal biomass can be seen as brown clusters around the plant root is highlighted in Figure 5A. The infrared image, Figure 5B, shows that a nitrate gradient exists, where reduced amounts of nitrate are seen further from the plant root and its primary fungal biomass.

To obtain a quantitative assessment of this nitrate gradient, the calibration curve in Figure 4B was used to calculate the nitrate concentration at each pixel. The line plot in Figure 5D shows how the nitrate concentration changes moving away from the plant root. Figure 5D shows that the nitrate concentration is at its lowest, 0 to $3 \mathrm{mM}$, within 1000 microns from the plant root, increases up to $35 \mathrm{mM}$ at 1500 microns and decreases almost steadily from $17 \mathrm{mM}$ at 3000 microns to $7 \mathrm{mM}$ at 5500 microns. This low concentration of $0-3 \mathrm{mM}$ nitrate within the first 1000 microns is likely due to the plant's uptake and consumption of the nitrate. The increased nitrate supply that surrounds this depleted region may be due to sequestration of nitrate by the fungi from the broader rhizosphere, hence a decrease in nitrate concentration exists following this available nutrient pool to the wider rhizosphere. Such pooling of high nitrate concentrations close to the root is in line with many other reports that indicate $\mathrm{N}$ sources are transferred from the fungi to the plant in exchange for carbon rich metabolites, such as simple 
hexoses derived from plant photosynthesis. ${ }^{20-22}$ The phenomenon is well characterized, but this is first report to visually characterize such activity at the chemical level.

In addition to nitrate, the sucrose concentration in the rhizosphere (Figure 5C) was also analyzed. Interestingly, Figure 5D shows that the sucrose concentration is low near the root, 3-6 $\mathrm{mM}$ within the first 1000 microns and increases further away from the root until it reaches to the concentration of the control media, $40 \mathrm{mM}$, at around 5500 microns. This pattern suggests that the sucrose, which is the carbon source in the media, is consumed by the fungi, hence the decrease in sucrose concentration in areas where the fungi is present.

\section{Discussion and conclusions}

Healthy soil is an important resource in both natural and agricultural ecosystems. It harbors both chemical and biological sources of nutrients that are required for plant growth, reproduction, insect and pathogen resistance, and response to changing environments. However, current agricultural practices rely heavily on the meticulous management of chemical nutrients. In natural ecosystems, nutrients in the rhizosphere are taken up by microorganisms like fungi and bacteria and are delivered to plants during interactions with plant roots in exchange for fixed carbon ${ }^{23,24}$. While such symbiotic relationships play an essential role in the regulation of soil nutrient cycling and subsequent carbon management, the complexity and variation of such natural systems has hindered the accurate assessment of the factors that maintain these important nutrient cycling interactions. This study is the first of its kind to visualize and quantify the nutrient availability and spatial location of key nutrient sources during such symbiotic associations. It thus represents a key step toward enhancing our understanding of the biological 
nutrient cycling pathways that are so essential to the development of both above- and belowground biomass.

Nitrogen is an essential macronutrient required for plant growth and reproduction, ${ }^{25}$ and its availability directly limits net primary productivity. ${ }^{19,26,27}$ Because plants in natural systems have a limited ability to utilize organic forms of nitrogen, the supply of nitrogen derived from the activity of the soil life is essential to biomass production. Nitrate is the primary form of nitrogen available to plants. ${ }^{28}$ By using FTIRI, the nitrate distribution in the poplar rhizosphere was mapped. The chemical images obtained from the rhizosphere provided both visual and quantitative evidence for the symbiotic sharing of nutrients between the plant and an ectomycorrhizal fungi, where the nitrate concentration was 5-fold higher around the fungal/root mantel, suggesting sequestration of nitrate from the media toward the plant root. The highest nitrate concentration was $35 \mathrm{mM}$ around 1500 microns away from the plant root, which then decreased to $7 \mathrm{mM}$ at 5500 microns, which is the baseline concentration in the media. Similarly, the sucrose used in the growth media as a carbon source was depleted to 3-6 $\mathrm{mM}$ around the mycorrhizal roots, suggesting its consumption by the fungus. It steadily increased to $\sim 40 \mathrm{mM}$ at 5500 microns away from the plant root. However, it should be noted that Laccaria does not possess the invertase enzyme that breaks sucrose into glucose and fructose. Therefore, it is likely that invertase is secreted from the plant roots into the media, thus facilitating breakdown of sucrose with subsequent uptake of glucose and fructose. ${ }^{29}$

Other studies have shown that carbon availability from the host triggers fungal nitrogen uptake and transport to plants during mychorrizal symbiosis. ${ }^{20-22,30}$ For example, Fellbaum et al. showed that an increase in carbon availability via the addition of $25 \mathrm{mM}$ sucrose to the root compartment increased the uptake of inorganic and organic nitrogen sources by the extra-radical 
mycelium (ERM) and transport to the root which resulted in two times as much nitrogen in the root tissue. ${ }^{30}$ This increase in nitrogen is facilitated by changes in fungal gene expression, including increases in the abundance of transcripts associated with nitrate transport. ${ }^{20,21}$ Studies also indicate that high affinity $\mathrm{N}$ transporters of fungi are highly expressed in the ERM but down regulated in the IRM which favors active absorption of nutrients like $\mathrm{NO}_{3}{ }^{-}$in the ERM, which plays a role in developing a strong concentration gradient across the mycorrhizal interface. ${ }^{20}$ This is consistent with the data presented here, which clearly demonstrates a nitrate gradient being formed surrounding the poplar - Laccaria mycorrhizal roots in the ERM. While significant progress has been made in understanding the $\mathrm{N}$ transport pathways of mycorrhizal fungi, it is still currently unknown in what form the nitrogen is transported from the fungal hyphae into the mycorrhizal Hartig net within the plant root cortex cells. Furthermore, the transport of other nutrients involved in such symbiotic interactions is not clear, and it is unknown how other nutrients except phosphorus, contribute to the carbon costs of symbiosis for the host plant. ${ }^{22}$

In this work, it was shown that FTIRI can reliably detect and quantify the nitrate and sucrose distribution in situ in the rhizosphere of poplar, which, with developing model systems, can help provide an understanding of the cost (carbon) to benefit (nutrient gain) ratio of symbiotic associations. The results showed evidence for symbiotic transport and sharing of nutrients between the plant and the ectomycorrhizal fungi, where the nitrate concentration was high around the root-associated fungi, suggesting sequestration from the media toward the plant root. Similarly, the sucrose used in the growth media as a carbon source was depleted around the fungi, suggesting its uptake and consumption.

In the future, this approach can be extended further to detect other essential inorganic nutrients including phosphates and sulfates. Its application is also suitable for studying other 
plant species or broader plant-microbe ecosystems that combine various plants, fungi, and bacteria while still having control of the system variables. Moreover, aided synchrotron infrared beams, subcellular analysis of the interface between the plant's root hairs and the rhizosphere can be pursued. The high brightness of a synchrotron source improves the spatial resolution to the diffraction limit of $2-10 \mu \mathrm{m}$ and improves spectral quality compared to a conventional globar source. In addition, it is possible to collect data more quickly and to oversample, which can improve the spatial resolution even further through deconvolution. ${ }^{31}$ By analyzing the plant's root hairs in the presence and absence of ectomycorrhizal fungi, a better understanding of the fungi's role in nutrient uptake in this symbiotic union will be possible. This ability to monitor nutrient changes with other microorganisms in the rhizosphere is a key step for understanding environmental nutrient flow processes.

With FTIRI providing a mechanism to probe how nature so effectively controls the availability of soil nutrients through the activity of beneficial microorganisms, future improvements may be made to land management programs that will allow more sustainable plant growth on an industrial scale by taking advantage of already existing plant-microbe relationships.

\section{Acknowledgements}

This work was supported by the United States Department of Energy, Office of Biological and Environmental Research, as part of the BER Genomic Science Program. This contribution originates primarily from grant number DE-SC0006652 (Leland Cseke, PI, UAH) as well as the "Environment Sensing and Response" Scientific Focus Area Program at Argonne National Laboratory. This research used resources of the NSLS and NSLS-II, U.S. Department 
of Energy (DOE) Office of Science User Facilities operated for the DOE Office of Science by Brookhaven National Laboratory under Contracts No. DE-AC02-98CH10886 and DESC0012704, respectively.

\section{Supporting Information Available:}

The supporting information provides additional details on the components of McCown Woody Plant medium and the methods used to determine the thickness of phytagel deposited on each slide. This material is available free of charge via the Internet at http://pubs.acs.org.

\section{References}

(1) O'Gara, F.; Dowling, D. N.; Boesten, B. Molecular Ecology of Rhizosphere Microorganisms: Biotechnology and the Release of GMOs; Wiley: New York, 2008.

(2) Zuccaro, A.; Lahrmann, U.; Langen, G. Curr Opinion Plant Biol 2014, 20, 135-145.

(3) Cruz, A. F.; Ishii, T. Biol Open 2012, 1, 52-57.

(4) Clode, P. L.; Kilburn, M. R.; Jones, D. L.; Stockdale, E. A.; Cliff, J. B.; Herrmann, A. M.; Murphy, D. V. Plant Phys 2009, 151, 1751-1757.

(5) Kilburn, M. R.; Jones, D. L.; Clode, P. L.; Cliff, J. B.; Stockdale, E. A.; Herrmann, A. M.; Murphy, D. V. Plant Signaling \& Behavior 2010, 5, 760-762.

(6) Lombi, E.; Scheckel, K. G.; Kempson, I. M. Environ Exper Botany 2011, 72, 3-17.

(7) Zhao, F.-J.; Moore, K. L.; Lombi, E.; Zhu, Y.-G. Trends Plant Sci 2014, 19, 183-192.

(8) Larsen, P. E.; Sreedasyam, A.; Trivedi, G.; Podila, G. K.; Cseke, L. J.; Collart, F. R. BMC Systems Biol 2011, 5, 1-14. 
(9) Larsen, P. E.; Trivedi, G.; Sreedasyam, A.; Lu, V.; Podila, G. K.; Collart, F. R. PLoS One 2010, 5, e9780.

(10) Lloyd, G.; McCown, B. Int. Plant Prop. Soc. Proc. 1981, 30, 421-427.

(11) Cseke, L. J.; Cseke, S. B.; Podila, G. K. Plant Cell Rep 2007, 26, 1529-1538.

(12) Park, J. K.; Khan, T.; Jung, J. Y. Carb Polymers 2006, 63, 482-486.

(13) Tewari, J.; Irudayaraj, J. J of Agr Food Chem 2004, 52, 3237-3243.

(14) Wang, J.; Kliks, M. M.; Jun, S.; Jackson, M.; Li, Q. X. J Food Sci 2010, 75, C208-C214.

(15) Brizuela, A. B.; Bichara, L. C.; Romano, E.; Yurquina, A.; Locatelli, S.; Brandán, S. A. Carb Res 2012, 361, 212-218.

(16) Goebbert, D. J.; Garand, E.; Wende, T.; Bergmann, R.; Meijer, G.; Asmis, K. R.; Neumark, D. M. J Phys Chem A 2009, 113, 7584-7592.

(17) Lane, M. D. Amer Mineralogist 2007, 92, 1-18.

(18) Kuhn, L. P. Anal. Chem. 1950, 22, 276-283.

(19) Hayat, R.; Ali, S.; Amara, U.; Khalid, R.; Ahmed, I. Ann Microbiol 2010, 60, 579-598.

(20) Ahmed, A. B. A.; Vakhrushev, A. V.; López, C.; Pogue, G. P.; Bücking, H.; Stangarlin, J.

R.; Malboobi, M. A.; Shahid, M. S.; Kamiński, P.; Mostafa, S. S. M. The Role of the Mycorrhizal Symbiosis in Nutrient Uptake of Plants and the Regulatory Mechanisms Underlying These Transport Processes; INTECH Open Access Publisher, 2012.

(21) Behie, S. W.; Bidochka, M. J. Trends in Plant Sci 2014, 19, 734-740.

(22) Bücking, H.; Kafle, A. Agronomy 2015, 5, 587-612.

(23) Vargas, W. A.; Mandawe, J. C.; Kenerley, C. M. Plant Physy 2009, 151, 792-808.

(24) Kraiser, T.; Gras, D. E.; Gutiérrez, A. G.; González, B.; Gutiérrez, R. A. J Exper Botany 2011, 62, 1455-1466. 
(25) Chrispeels MJ, C. N., Schroeder JI. The Plant Cell 1999, 11, 661-676.

(26) Richardson, A.; Barea, J.-M.; McNeill, A.; Prigent-Combaret, C. Plant and Soil 2009, 321, 305-339.

(27) Chamam, A.; Sanguin, H.; Bellvert, F.; Meiffren, G.; Comte, G.; Wisniewski-Dyé, F.; Bertrand, C.; Prigent-Combaret, C. Phytochem 2013, 87, 65-77.

(28) Miller, A. J. In Encyclopedia of Life Sciences; John Wiley \& Sons: Chichester, 2010.

(29) Nehls, U.; Göhringer, F.; Wittulsky, S.; Dietz, S. Plant Biol 2010, 12, 292-301.

(30) Fellbaum, C. R.; Gachomo, E. W.; Beesetty, Y.; Choudhari, S.; Strahan, G. D.; Pfeffer, P.

E.; Kiers, E. T.; Bücking, H. Proc Nat Acadf Sci 2012, 109, 2666-2671.

(31) Stavitski, E.; Smith, R. J.; Bourassa, M. W.; Acerbo, A. S.; Carr, G. L.; Miller, L. M. Anal. Chem. 2013, 85, 3599-3605.

\section{Figure Captions}

Figure 1. (A) Slide preparation for growing Populus tremuloides. A sterile chilled reflective glass slide was immersed into a $50^{\circ} \mathrm{C}$ WPM + Phytagel solution. As the slide is pulled from the solution, a layer of media deposits over the slide which becomes a thin gel $(\sim 0.5 \mathrm{~mm})$ as the Phytagel solidifies. A poplar seedling is then embedded into the gelled media at the top end of the slide and is allowed to grow for 5-7 weeks either with or without fungal interaction. (B) Populus tremuloides grown on an IR-reflective glass slide in a conical tube. The image shows how the plant roots grow vertically downwards on the slide. The seedlings receive nutrients from the Woody Plant Media (WPM) which facilitates the plant growth and interaction with Laccaria. 
Figure 2. FTIR spectra of the components in the plant media: Phytagel (green), Woody Plant Medium (WPM) (blue) and sucrose (red). In the Phytagel spectrum, the carboxylate ester peak at $1610 \mathrm{~cm}^{-1}$ and polysaccharide peaks $\left(1000-1200 \mathrm{~cm}^{-1}\right)$ are most prominent. The glycosidic bond in sucrose is present at $925 \mathrm{~cm}^{-1}$. Primary nutrients (nitrates, phosphates, sulfates) are found in WPM and have vibrational modes between $1300-1500 \mathrm{~cm}^{-1}$. WPM has a negligible contribution compared to other components in the media.

Figure 3. FTIR absorption spectra of ${ }^{14} \mathrm{NH}_{4}{ }^{14} \mathrm{NO}_{3},{ }^{14} \mathrm{NH}_{4}{ }^{15} \mathrm{NO}_{3}$, and ${ }^{15} \mathrm{NH}_{4}{ }^{14} \mathrm{NO}_{3}$ showing the ${ }^{14} \mathrm{NH}_{4}{ }^{+}$bending vibration at $1432 \mathrm{~cm}^{-1}$ and ${ }^{14} \mathrm{NO}_{3}{ }^{-}$stretching vibration mode at $1340 \mathrm{~cm}^{-1}$. The inset shows the ${ }^{14} \mathrm{NO}_{3}{ }^{-}$bending mode at $827 \mathrm{~cm}^{-1}$. Upon isotopic substitution, the ${ }^{15} \mathrm{NH}_{4}{ }^{+}$bending vibration shifts to $1425 \mathrm{~cm}^{-1}$. The ${ }^{15} \mathrm{NO}_{3}{ }^{-}$stretching vibration and the ${ }^{15} \mathrm{NO}_{3}$ bending vibration shift to 1305 and $806 \mathrm{~cm}^{-1}$, respectively.

Figure 4. (A) FTIR absorption spectra of the Phytagel media with increasing concentrations of ammonium nitrate at 7, 10, 15, and $20 \mathrm{mM}$. (B) Calibration curve of nitrate absorbance at 1340 $\mathrm{cm}^{-1}$ vs. nitrate concentration. The line plot shows a direct linear correlation between nitrate absorbance and concentration.

Figure 5. (A) Visible image of a Poplar plant root embedded in Phytagel media. Brown regions represent fungal biomass. The image shows fungal colonization over the plant root. (B) FTIR image of the same sample showing the distribution of nitrate in the rhizosphere. The image shows that the nitrate concentration is elevated in the regions of high fungal colonization and 
decreases away from the plant root as the fungal population decreases. (C) FTIR image showing distribution of sucrose in the rhizosphere. The plot shows that the sucrose concentration increases away from the plant root as the fungal population decreases. (D) Plot of nitrate and sucrose concentration versus distance from the poplar plant root. Points on the plot were extracted from the line profiles in Figures 5B and 5C for nitrate and sucrose, respectively. The trend suggests that there is symbiotic sharing of nutrients between the plant and the fungi where the fungi consumes the sucrose and sequesters nitrate for the plant's use. Scale bar is $1 \mathrm{~mm}$ for all images. 


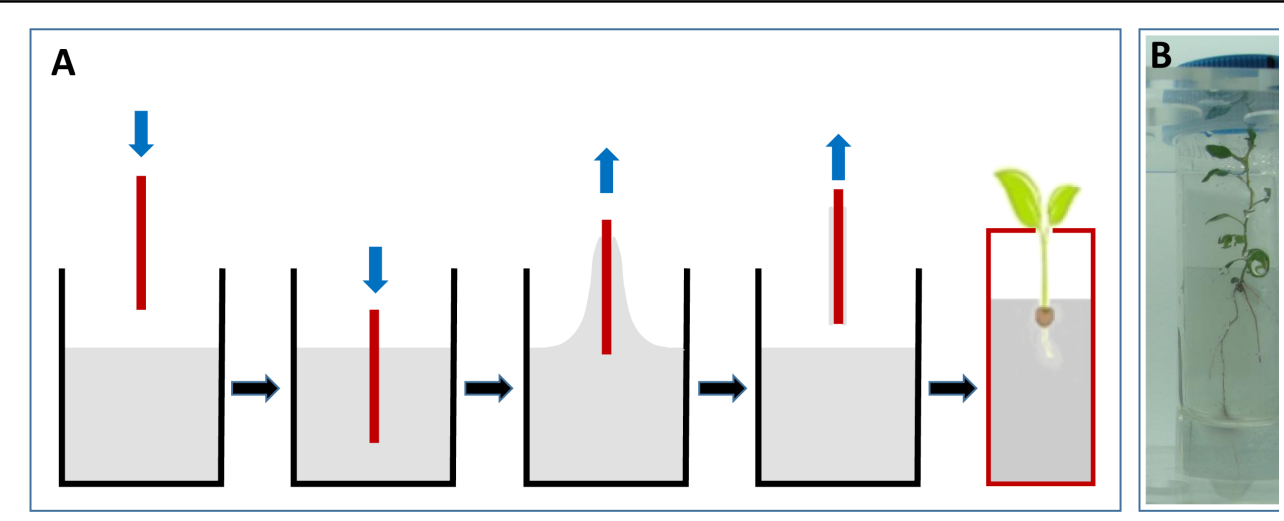

Figure 1. (A) Slide preparation for growing Populus tremuloides. A sterile chilled reflective glass slide was immersed into a $50^{\circ} \mathrm{C}$ WPM + Phytagel solution. As the slide is pulled from the solution, a layer of media deposits over the slide which becomes a thin gel $(\sim 0.5 \mathrm{~mm})$ as the Phytagel solidifies. A poplar seedling is then embedded into the gelled media at the top end of the slide and is allowed to grow for 5-7 weeks either with or without fungal interaction. (B) Populus tremuloides grown on an IR-reflective glass slide in a conical tube. The image shows how the plant roots grow vertically downwards on the slide. The seedlings receive nutrients from the Woody Plant Media (WPM) which facilitates the plant growth and interaction with Laccaria. 


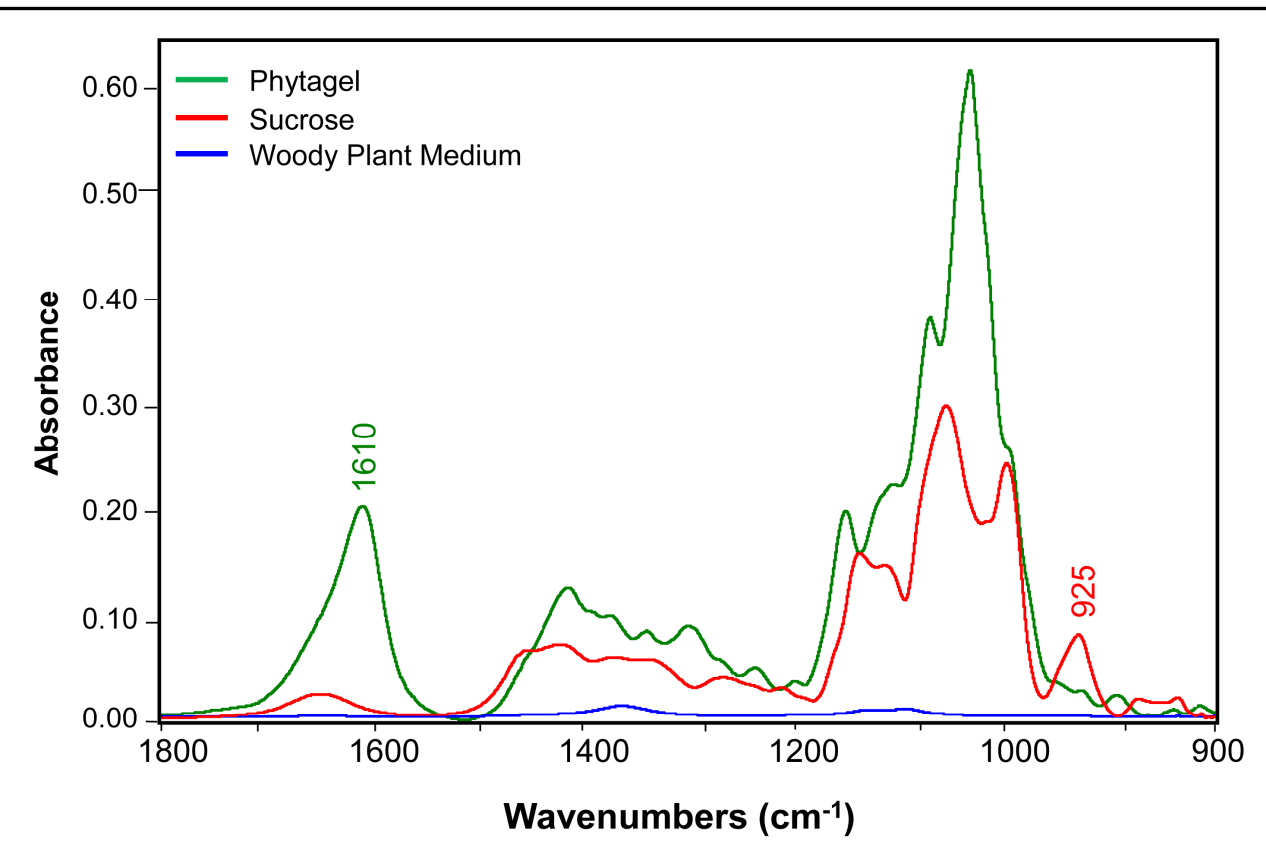

Figure 2. FTIR spectra of the components in the plant media: Phytagel (green), Woody Plant Medium (WPM) (blue) and sucrose (red). In the Phytagel spectrum, the carboxylate ester peak at $1610 \mathrm{~cm}^{-1}$ and polysaccharide peaks $\left(1000-1200 \mathrm{~cm}^{-1}\right)$ are most prominent. The glycosidic bond in sucrose is present at $925 \mathrm{~cm}^{-1}$. Primary nutrients (nitrates, phosphates, sulfates) are found in WPM and have vibrational modes between 1300-1500 $\mathrm{cm}^{-1}$. WPM has a negligible contribution compared to other components in the media. 

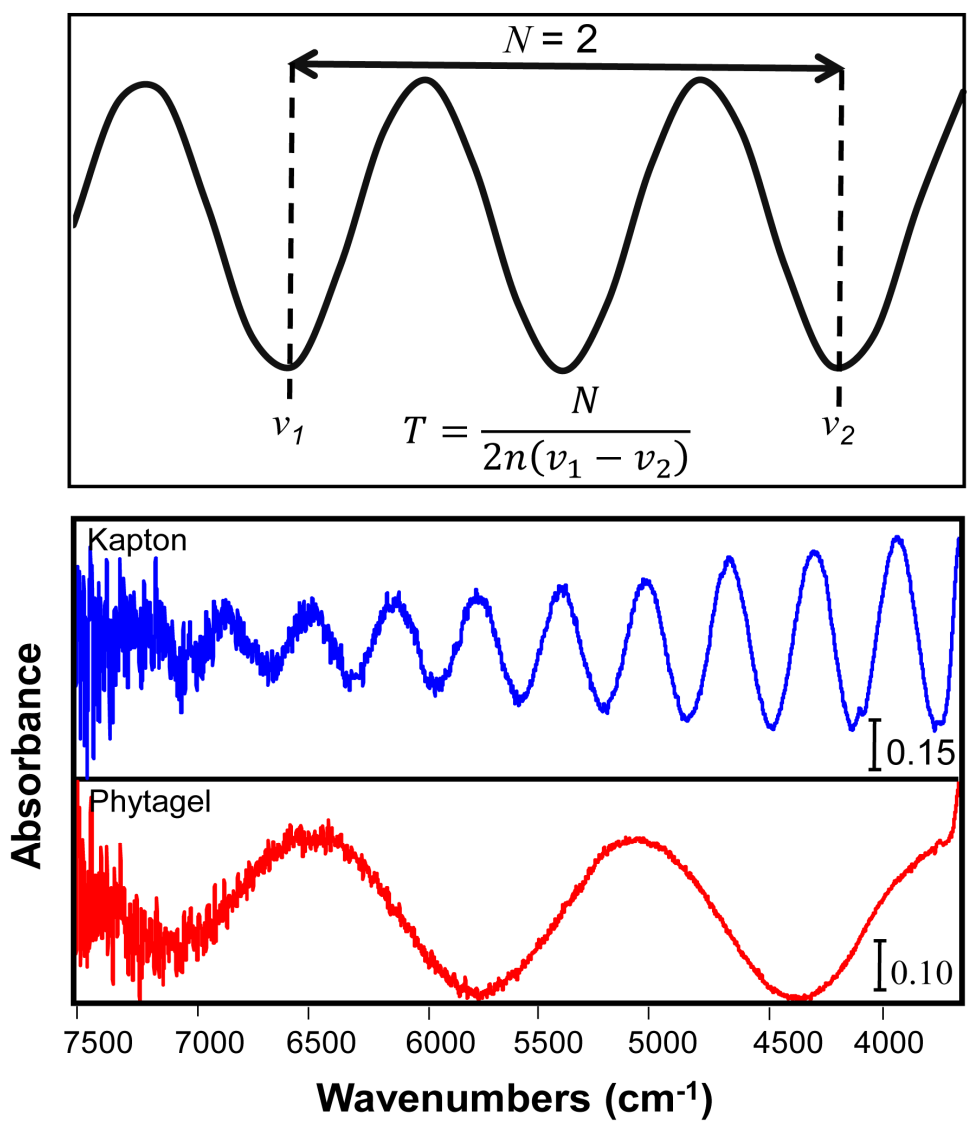

Figure 3. FTIR absorption spectra of ${ }^{14} \mathrm{NH}_{4}{ }^{14} \mathrm{NO}_{3},{ }^{14} \mathrm{NH}_{4}{ }^{15} \mathrm{NO}_{3}$, and ${ }^{15} \mathrm{NH}_{4}{ }^{14} \mathrm{NO}_{3}$ showing the ${ }^{14} \mathrm{NH}_{4}{ }^{+}$bending vibration at $1432 \mathrm{~cm}^{-1}$ and ${ }^{14} \mathrm{NO}_{3}{ }^{-}$stretching vibration mode at $1340 \mathrm{~cm}^{-1}$. The inset shows the ${ }^{14} \mathrm{NO}_{3}{ }^{-}$bending mode at $827 \mathrm{~cm}^{-1}$. Upon isotopic substitution, the ${ }^{15} \mathrm{NH}_{4}{ }^{+}$bending vibration shifts to $1425 \mathrm{~cm}^{-1}$. The ${ }^{15} \mathrm{NO}_{3}{ }^{-}$ stretching vibration and the ${ }^{15} \mathrm{NO}_{3}$ bending vibration shift to 1305 and $806 \mathrm{~cm}^{-1}$, respectively. 


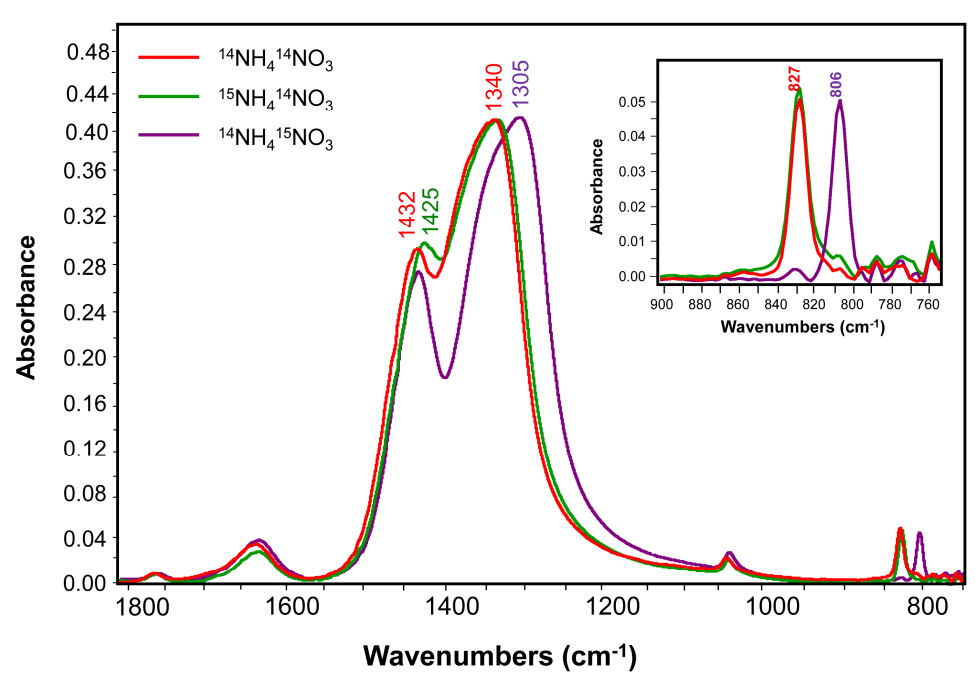

Figure 4. (A) FTIR absorption spectra of the Phytagel media with increasing concentrations of ammonium nitrate at 7, 10, 15, and $20 \mathrm{mM}$. (B) Calibration curve of nitrate absorbance at $1340 \mathrm{~cm}^{-1} v s$. nitrate concentration. The line plot shows a direct linear correlation between nitrate absorbance and concentration. 

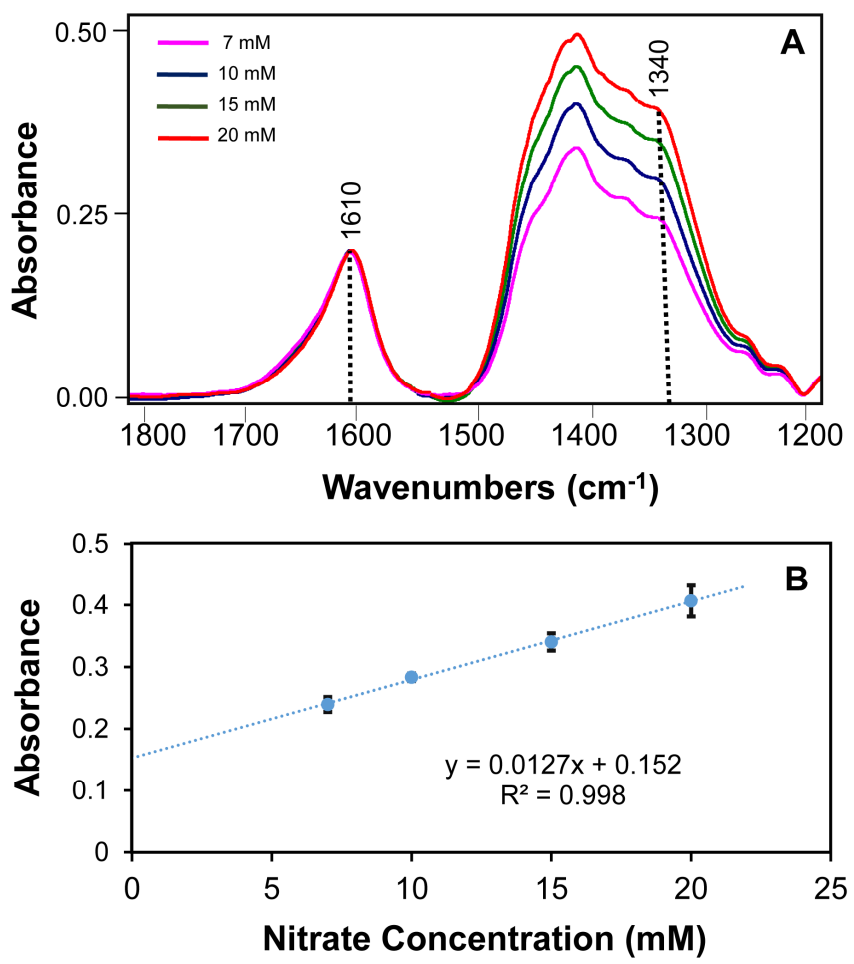

Figure 5. (A) Visible image of a Poplar plant root embedded in Phytagel media. Brown regions represent fungal biomass. The image shows fungal colonization over the plant root. (B) FTIR image of the same sample showing the distribution of nitrate in the rhizosphere. The image shows that the nitrate concentration is elevated in the regions of high fungal colonization and decreases away from the plant root as the fungal population decreases. (C) FTIR image showing distribution of sucrose in the rhizosphere. The plot shows that the sucrose concentration increases away from the plant root as the fungal population decreases. (D) Plot of nitrate and sucrose concentration versus distance from the poplar plant root. Points on the plot were extracted from the line profiles in Figures $\mathbf{5 B}$ and $\mathbf{5 C}$ for nitrate and sucrose, respectively. The trend suggests that there is symbiotic sharing of nutrients between the plant and the fungi where the fungi consumes the sucrose and sequesters nitrate for the plant's use. Scale bar is $1 \mathrm{~mm}$ for all images. 


\section{SUPPLEMENTAL INFORMATION}

\section{Imaging Nutrient Distribution in the Rhizosphere using FTIR Imaging}

Tiffany Victor, ${ }^{1}$ Natalie Delpratt, ${ }^{2}$ Sarah Beth Cseke, ${ }^{3}$ Lisa M. Miller, ${ }^{1,2}$ Leland James Cseke ${ }^{3 *}$

${ }^{1}$ Department of Chemistry, Stony Brook University, Stony Brook, New York 11794

${ }^{2}$ National Synchrotron Light Source II, Brookhaven National Laboratory, Upton, New York 11973

${ }^{3}$ Department of Biological Science, University of Alabama in Huntsville, Huntsville, Alabama 35899

${ }^{*}$ Corresponding author:

Leland J. Cseke, PhD

Department of Biological Science

The University of Alabama in Huntsville

Huntsville, Alabama 35899

Phone: (256) 824-6774

Fax: (256) 824-6305

E-mail: csekel@uah.edu

This supplemental information presents additional information on the components of McCown Woody Plant medium and the methods used to determine the thickness of phytagel deposited on each slide. 
Determination of the phytagel thickness using the fringing effect

The concentration of nitrate in the poplar rhizosphere was determined using the BeerLambert Law $(\mathrm{A}=\varepsilon b c)$, where $\mathrm{A}$ is the FTIR absorbance at a particular wavelength, $\varepsilon$ is the absorption coefficient at a particular wavelength, $\mathrm{b}$ is the sample thickness, and $\mathrm{c}$ is the sample concentration. However, as is noted in the Beer-Lambert Law, knowledge of the absorption coefficient and sample thickness must be known in order to determine the nutrient concentration. To calculate the Phytagel thickness, the fringing effect of thin films was applied. To do so, the Phytagel matrix was dried down to reduce sample thickness and to remove the strong, broad absorption bands of water that would contribute to the spectra. The dried Phytagel film was placed into a FTIR sandwich cell with a $13 \mathrm{~mm}^{2}$ round opening and five FTIR spectra were collected from different regions in transmission mode from $7000-750 \mathrm{~cm}^{-1}$, using a spectral resolution of $4 \mathrm{~cm}^{-1}$ and 128 scans per spectrum. A similar procedure was followed for a $8 \mu \mathrm{m}-$ thick Kapton (polyimide) film as a control. To calculate the Phytagel thickness, the fringing effect of thin films was applied using the following equation:

$$
\mathrm{b}=\mathrm{N} / 2 \mathrm{n}\left(\mathrm{v}_{1}-\mathrm{v}_{2}\right)
$$

where $\mathrm{b}=$ path length of the cell, $\mathrm{n}=$ the refractive index of the material, $\mathrm{N}=$ the number of fringes within a given spectral region, and $v_{1}$ and $v_{2}$ are the start and end points of that spectral region.

The fringing effect from the Kapton polyimide film, used as a reference to determine the accuracy of this method, revealed 3 fringes in the spectral region from $6660-5560 \mathrm{~cm}^{-1}$ (Figure S1). Using its refractive index of 1.7 , the calculated thickness was $8.2 \pm 0.2 \mu \mathrm{m}$, which was very 
close to the manufacturer's reported thickness of $8 \mu \mathrm{m}$. For the Phytagel film, 2 fringes were observed in the spectral region from $7075-4345 \mathrm{~cm}^{-1}$. We estimated a refractive index of 1.5 for Phytagel based on the refractive index of other polymers in its class such as poly vinyl alcohol, which resulted in a calculated thickness of $2.4 \pm 0.07 \mu \mathrm{m}$. The error of the technique was determined based on the $3 \%$ error obtained from the polyimide film.

Figure S-1. Interference fringes in the FTIR transmission spectra of free standing films of Kapton polyimide (blue) and Phytagel (red). The fringing effect was used to calculate the thickness of the dried Phytagel film, which was found to be $2.4 \mu \mathrm{m}$. An example of a Kapton polyimide reference film is also shown. The refractive index used in calculating the film thickness was 1.7 and 1.5 for polyimide and Phytagel respectively. 
Table S1. Composition of nutrient medium used for growing plants

\begin{tabular}{lc}
\hline Component & Concentration (mM) \\
\hline Phytagel & 0.008 \\
Sucrose & 40 \\
MES (2-ethanesulfonic acid) & 2.6 \\
& \\
Woody Plant Medium & \\
Ammonium nitrate & 5.0 \\
Potassium sulfate & 5.0 \\
Magnesium sulfate & 2.0 \\
Calcium nitrate & 2.0 \\
Potassium Phosphate & 1.0 \\
Calcium chloride & 0.6 \\
Copper Sulfate & 0.001 \\
EDTA & 0.1 \\
Boric acid & 0.1 \\
Magnesium sulfate & 0.1 \\
Molybdic acid & 0.002 \\
Ferrous sulfate & 0.1 \\
Zinc sulfate & 0.03 \\
Myo-Inositol & 0.5 \\
Glycine & 0.03 \\
Thiamine HCl & 0.006 \\
Nicotinic Acid & 0.005 \\
Pyridoxine HCl & 0.002 \\
\hline
\end{tabular}

